\title{
Carathéodory-type Theorems à la Bárány
}

\author{
Jürgen Bokowski · Javier Bracho • Ricardo Strausz
}

Received: 26 December 2009 / Revised: 29 July 2010 / Accepted: 25 August 2010 /

Published online: 11 September 2010

(C) Springer Science+Business Media, LLC 2010

\begin{abstract}
We generalise the famous Helly-Lovász theorem leading to a generalisation of the Bárány-Carathéodory theorem for oriented matroids in dimension $\leq 3$. We also provide a non-metric proof of the latter colourful theorem for arbitrary dimensions and explore some generalisations in dimension 2.
\end{abstract}

Keywords Helly-type theorems · Carathéodory-type theorems · Colourful theorems · Abstract convexity · Oriented Matroids · Separoids

\section{Introduction}

In 1907 Constantin Carathéodory proved the following corner-stone of combinatorial convexity.

Theorem 1 (Carathéodory [8]) A point $\mathbf{a} \in \mathbb{E}^{d}$ lies in the convex hull of a given subset $B \subset \mathbb{E}^{d}$ of d-dimensional Euclidean space if and only if it is the convex hull

The present work was done while the first author had a sabbatical position at the Instituto de Matemáticas, Universidad Nacional Autónoma de México.

J. Bokowski $(\bowtie) \cdot$ J. Bracho · R. Strausz

Instituto de Matemáticas, Universidad Nacional Autónoma de México, Ciudad Universitaria 04510,

Mexico DF, Mexico

e-mail: juergen@bokowski.de

J. Bracho

e-mail: roli@math.unam.mx

R. Strausz

e-mail: dino@math.unam.mx

J. Bokowski · J. Bracho · R. Strausz

www.math.unam.mx 
of $d+1$ elements of $B$; in symbols,

$$
\mathbf{a} \dagger B \quad \Longleftrightarrow \quad \exists B^{\prime} \in\left(\begin{array}{c}
B \\
d+1
\end{array}\right): \mathbf{a} \dagger B^{\prime} .
$$

We denote by $A \dagger B$ the fact that $A$ is not separated from $B$, i.e., $\operatorname{conv}(A) \cap$ $\operatorname{conv}(B) \neq \emptyset$ (while $A \cap B=\emptyset$; cf. [16]). If we abbreviate $[k]:=\{1,2, \ldots, k\}$ and write simply $\mathbf{b}_{1} \mathbf{b}_{2} \cdots \mathbf{b}_{d}$ instead of $\left\{\mathbf{b}_{1}, \mathbf{b}_{2}, \ldots, \mathbf{b}_{d}\right\}$, a beautiful and deep generalisation of Carathéodory's theorem, due to Imre Bárány (1982), can be written as follows.

Theorem 2 (Bárány [4, Theorem 2.1]) Let $B_{1}, B_{2}, \ldots, B_{d+1} \subset \mathbb{E}^{d}$ and $\mathbf{a} \in \mathbb{E}^{d}$. Iffor each $i \in[d+1]$ we have that $\mathbf{a} \dagger B_{i}$, then there exists a choice $\mathbf{b}_{i} \in B_{i}$, for $i \in[d+1]$, such that $\mathbf{a} \dagger \mathbf{b}_{1} \mathbf{b}_{2} \cdots \mathbf{b}_{d+1}$.

Observe that if all $B_{i}$ are equal, we are back at Carathéodory's theorem. The sets $B_{i}$ are referred as colours and this result is also known as "colourful Carathéodory theorem".

A stronger version was proved in the same paper of Bárány; namely, not all colours need to capture the given point, it suffices that all but one capture the point:

Theorem 3 (Bárány [4, Theorem 2.3]) Let $B_{1}, B_{2}, \ldots, B_{d} \subset \mathbb{E}^{d}$ and $\mathbf{a}, \mathbf{b} \in \mathbb{E}^{d}$. Iffor each $i \in[d]$ we have that $\mathbf{a} \dagger B_{i}$, then there exists a choice $\mathbf{b}_{i} \in B_{i}$, for $i \in[d]$, such that $\mathbf{a} \dagger \mathbf{b b}_{1} \mathbf{b}_{2} \cdots \mathbf{b}_{d}$.

This strengthening can be rephrased as follows: if each heterochromatic simplex is separated from the origin, then there are two colours which are separated from the origin. More recently, two independent groups have observed that these hypotheses can be weakened a bit more; namely, it suffices that the union of pairs of colours captures the point:

Theorem 4 (Arocha et al. [1], Holmsen et al. [11]) Let $B_{1}, B_{2}, \ldots, B_{d+1} \subset \mathbb{E}^{d}$ and $\mathbf{a} \in \mathbb{E}^{d}$. If for each $i, j \in[d+1], i \neq j$, we have that $\mathbf{a} \dagger\left(B_{i} \cup B_{j}\right)$, then there exists a choice $\mathbf{b}_{i} \in B_{i}$, for $i \in[d]$, such that $\mathbf{a} \dagger \mathbf{b}_{1} \mathbf{b}_{2} \cdots \mathbf{b}_{d+1}$.

This further strengthening can be rephrased as follows: if each heterochromatic simplex is separated from the origin, then there are two colours whose union is separated from the origin. A couple of questions arise: How far can the hypothesis of Theorem 2 be weakened without changing the conclusion? Is there a purely combinatorial Bárány-Carathéodory theorem?

In Sect. 2 we provide yet another proof of Theorem 3; this emphasises the nonmetric aspects of it and how the linear order of $\mathbb{R}$ is playing the main role. In Sect. 3 we generalise Theorem 3 to the combinatorial settings of oriented matroids-even though we had to restrict ourselves to dimension 3. Finally, in Sect. 4 we explore two more directions, in dimension 2, where the hypothesis can be weakened; namely, changing points to convex sets and relaxing the condition on the colouring, to consider all colourings, while restricting "the position" of the points in the configuration. 
In all cases, the conclusion is the same: there exists a heterochromatic simplex "capturing" a point.

To settle Theorem 3 for oriented matroids in dimension 3, we also generalise the colourful version of Helly's theorem for hemispaces in the language of rank 3 oriented matroids (see also [3]). We will use the topological representation theorem due to Jon Folkman and Jim Lawrence [9]. For, let $\mathbf{r}_{1}, \ldots, \mathbf{r}_{4} \in \mathbb{E}^{3}$ be the vertices of a (red) tetrahedron that contains the origin $\mathbf{0}$ in its interior (i.e., $\mathbf{0} \dagger \mathbf{r}_{1} \ldots \mathbf{r}_{4}$ ). Now, let $r_{i}=\mathbf{r}_{i}^{\perp} \cap \mathbb{S}^{2}$ be the great circle in the unit sphere centred at $\mathbf{0}$ whose spanned plane is orthogonal to $\mathbf{r}_{i}$, for $i \in$ [4], and let each such circle be oriented in such a way that it "remembers" the side where the vector $\mathbf{r}_{i}$ is pointing at-we call that side the positive hemisphere related to $r_{i}$. Observe that the fact that the origin is not separated from those 4 vectors is equivalent to the fact that the intersection of their positive hemispheres is empty. It is easy to see that these four oriented circles represent, in the sense of Folkman and Lawrence (see also [5]), the unique rank 3 cyclic oriented matroid on four elements; we denote this oriented matroid by $\Delta_{4}$. If we do the same with the other two tetrahedra (the green and the blue), we arrive at the hypothesis of the strong version of Bárány's theorem; however, if we now allow the great circles to "wiggle a little bit" by a topological transformation-to become a configuration of pseudolines in the sphere-we arrive at an oriented matroid (and, indeed, all oriented matroids of rank 3 can be represented in such a way). In the language of oriented matroids, we prove the following:

Main Theorem Let $M=(E, \mathcal{C})$ be a rank 3 oriented matroid with $|E|=12$ elements, endowed with a three-colouring $E=R \cup G \cup B$ such that each colour class consists of four elements. Furthermore, suppose that the restriction of $M$ to each colour class is isomorphic to $\Delta_{4}$-the (unique) rank 3 cyclic oriented matroid on four elements. Then, for every single element extension $E \cup y$, there exists an element in each of the colour classes $r \in R, g \in G, b \in B$ such that the restriction to the elements $\{r, g, b, y\}$ is isomorphic to $\Delta_{4}$.

Since there are some millions of non-stretchable rank 3 oriented matroids with 12 elements, this is truly a generalisation (recall that the smallest example of a uniform non-stretchable oriented matroid in rank 3, the so-called non-pappus configuration due to Gerhard Ringel, has nine elements; see [6]).

Indeed, we believe that the result should follow in arbitrary dimension, but the techniques we develop here-as will be noted in situ-do not seem to be sufficient to solve the problem. We propose here the following

Conjecture The Bárány-Carathéodory theorem is true for all oriented matroids.

\section{A Non-metric Proof of the Bárány-Carathédory Theorem 3}

In order to prove Theorem 3, let us reinterpret what does it means to "trap the origin" in terms of "covering the sphere". We restrict ourselves to the general position case (the general case is just a bit more obscure and adds nothing to the 
context). Observe that if $B \cup\{\boldsymbol{0}\}$ is in general position, then $\mathbf{0} \dagger B$ if and only if $\operatorname{pos}(B)=\mathbb{R}^{d}$, where $\operatorname{pos}(\cdot)$ denotes the positive span closure operator. Also, since we want to avoid metrical properties of Euclidian space, we will work in the "topological" sphere.

Let $\mathbb{R}^{d}$ be the real $d$-dimensional vector space over the totally ordered field $(\mathbb{R},<)$, and let

$$
\mathbb{S}^{d-1}:=\left(\mathbb{R}^{d} \backslash\{\mathbf{0}\}\right) / \mathbb{R}^{+}
$$

be "the sphere" centred at the origin (where $\mathbb{R}^{+}=\{x \in \mathbb{R}: 0<x\}$ denotes the set of positive scalars). Now, let $P=\bigcup_{i \in[d]} P_{i} \subset \mathbb{S}^{d-1}$ be a coloured subset such that $\mathbf{0} \dagger P_{i}$. Finally, let $\mathbf{y} \in \mathbb{S}^{d-1}$ be any other point. In order to prove that there is a heterochromatic choice $\mathbf{x}_{i} \in P_{i}$ such that $\mathbf{0} \dagger \mathbf{x}_{1} \ldots \mathbf{x}_{d} \mathbf{y}$, it is enough to prove that

$$
\mathbb{S}^{d-1}=\bigcup_{\mathbf{x}_{i} \in P_{i}} \operatorname{pos}\left(\mathbf{x}_{1}, \ldots, \mathbf{x}_{d}\right),
$$

where the union is taken over all heterochromatic choices-simply observe that $\mathbf{0} \dagger \mathbf{x}_{1} \ldots \mathbf{x}_{d} \mathbf{y}$ if and only if $-\mathbf{y} \in \operatorname{pos}\left(\mathbf{x}_{1}, \ldots, \mathbf{x}_{d}\right)$.

That is, we want to prove that if the union of simplices of each colour cover the sphere, then the union of heterochromatic simplices also cover the sphere. For this purpose, suppose this is not true and let

$$
\mathbf{b} \in B:=\partial \bigcup_{\mathbf{x}_{i} \in P_{i}} \operatorname{pos}\left(\mathbf{x}_{1}, \ldots, \mathbf{x}_{d}\right)
$$

be a point in the boundary of such a union. Clearly, such a point is in the positive cone of some heterochromatic subset of (at most) $d-1$ elements, say $\mathbf{b} \in$ $\operatorname{pos}\left(\mathbf{x}_{1}, \ldots, \mathbf{x}_{d-1}\right)$, where $\mathbf{x}_{i} \in P_{i}$ for each $i \in[d-1]$. Then the hypersphere $H:=$ $\operatorname{span}\left(\mathbf{x}_{1}, \ldots, \mathbf{x}_{d-1}\right)$ leaves all points of $P_{d}$, the last colour, in one of the hemispheres it defines; this contradicts the hypothesis $\mathbf{0} \dagger P_{d}$.

\section{Proof of Main Theorem}

An oriented matroid can be defined as a Radon-Steinitz separoid (see e.g. [16]). In order to prove our main theorem, we first reduce the problem-just as we will do in Proposition 8-using the so-called Steinitz exchange axiom (see Fig. 4); namely, for each minimal Radon partition $A \dagger B$, if $c \notin(A \cup B)$ then there exists $d \in(A \cup B)$ such that $(A \backslash d) \dagger(B \backslash d \cup c)$. So, instead of considering four red oriented pseudolines, four blue and four green, each set with the property that their hemiplanes have empty intersection (they are isomorphic to $\Delta_{4}$ ), we add the 13th element in yellow (which plays the role of $c$ above) and drop a red, a blue and a green element by using, three times, Steinitz exchange property (see Fig. 1). That reduces the problem of examining all configurations with 13 pseudolines to those with only ten of them. 
Fig. 1 (Color online)

A four-coloured rank 3 oriented matroid with ten elements

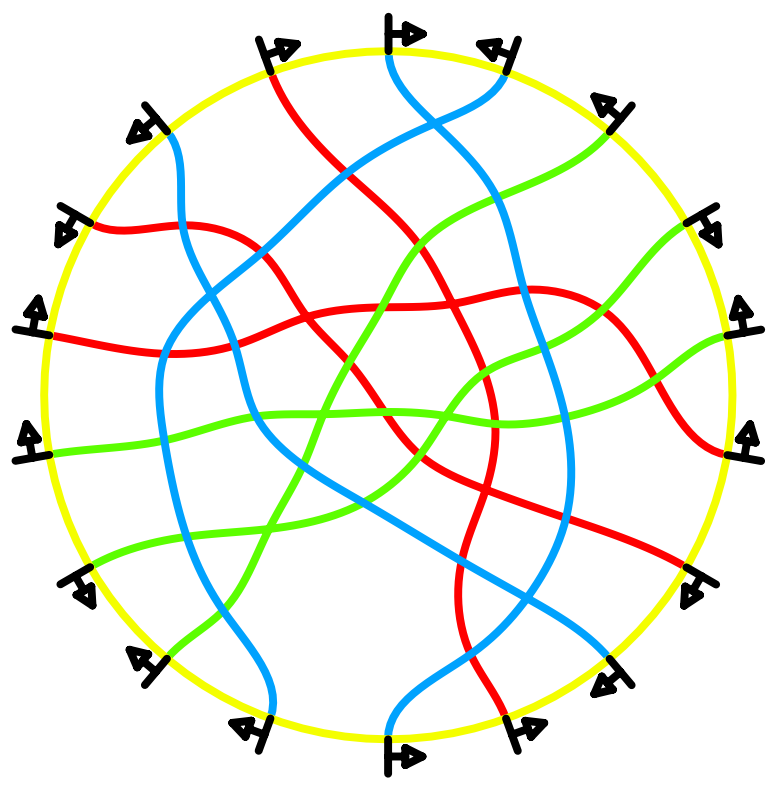

\subsection{Three Guards Instead of Nine Suffice; A Guard Problem}

We consider an island in form of a circular disc.

We consider three times three different pairs of antipodal points on the shore of this island. These pairs of antipodal points are connected by one-sided defence walls. Say, we have three red, three green, and three blue such defence walls, each set of three such walls with equal colours belonging to a group of red, green, and blue people, respectively.

A triangle formed by three defence walls will be called a defendable triangle, when the defence walls are all directed towards the outside of this triangle. We assume that the three sets of three walls each, one set for each given colour, each form defendable triangles. In other words, each group of a given color can hide a treasure inside a corresponding defendable triangle formed by defence walls of the corresponding colour.

Given any such system of nine defence walls on a circular island, can we find a defendable rainbow triangle, i.e., one defendable triangle that consist of a red, a green, and a blue side?

This would mean that the three groups can move their treasures to this rainbow triangle and instead of using all nine defence walls, they can use only three of them, one of each group.

We answer affirmatively the above question.

Theorem 5 The existence of a defendable triangle for each triple of defence walls with equal colors implies the existence of a defendable rainbow triangle.

For the stretchable case, the theorem is simply coloured Helly for hemispaces: Given three families of convex sets, say the reds, the blues and the greens, if, when- 
ever we take a colorful triple, they have a common point, then there is a color and a point in common to all convex sets of that color. Conversely, if no monochromatic family has a common point, there is a colorful triple without a point in common. We generalise this to pseudohemispaces of an oriented matroid in rank 3 using the following result, due to Friedrich Wilhelm Levi [12], published in the first paper on pseudoline arrangements (see also [10]).

Lemma 6 (Levi's enlargement lemma) For every two non-colinear points in an arrangement of pseudolines, there is a one element extension that goes through these points.

A direct consequence of Levi's enlargement lemma is the following result.

Lemma 7 It suffices to prove our theorem for those arrangements of pseudolines for which the three defendable triangles with a given color are not intersected by any other pseudoline.

Proof Assume w.l.o.g. that in the given arrangement a non-red pseudoline intersects the red triangle. We are going to use Levi's enlargement lemma. We pick the first point as the intersection of one arbitrary red pseudoline $r$ with the line at infinity. We pick the second point as the intersection of the two remaining red pseudolines. The resulting pseudoline $\ell$ by Levi's enlargement lemma does not intersect the chosen red pseudoline $r$ in an additional point (different from the first point). We replace the chosen red pseudoline $r$ with the new pseudoline $\ell$, however, we use a small local change at the intersection point of all three red pseudolines so that a defendable triangle occurs. It is clear how the new pseudoline has to be oriented. When we have proved our theorem for the arrangement with the resulting pseudoline arrangement, we see that it also implies the existence of a defendable rainbow triangle for the original arrangement. This rainbow triangle will be even greater than the one for which we prove the theorem.

Figure 2 shows the situation after applying the above lemma (cf. with Fig. 1).

The absence of an enlargement lemma for higher dimensions is an obstruction to prove the conjecture.

\subsection{Case Distinction and Main Part of the Proof}

We consider, say with a yellow element at infinity, a red triangle that does not touch the line at infinity as well as a blue triangle that does not touch the line at infinity either.

The three supporting pseudolines of these two topological triangles each define together with the line at infinity either triangular regions or topological 4-gons along the line at infinity. In one of these regions (triangle or 4-gon) the corresponding other triangle has to lie. 
Fig. 2 (Color online) No pseudoline intersects a defendable triangle

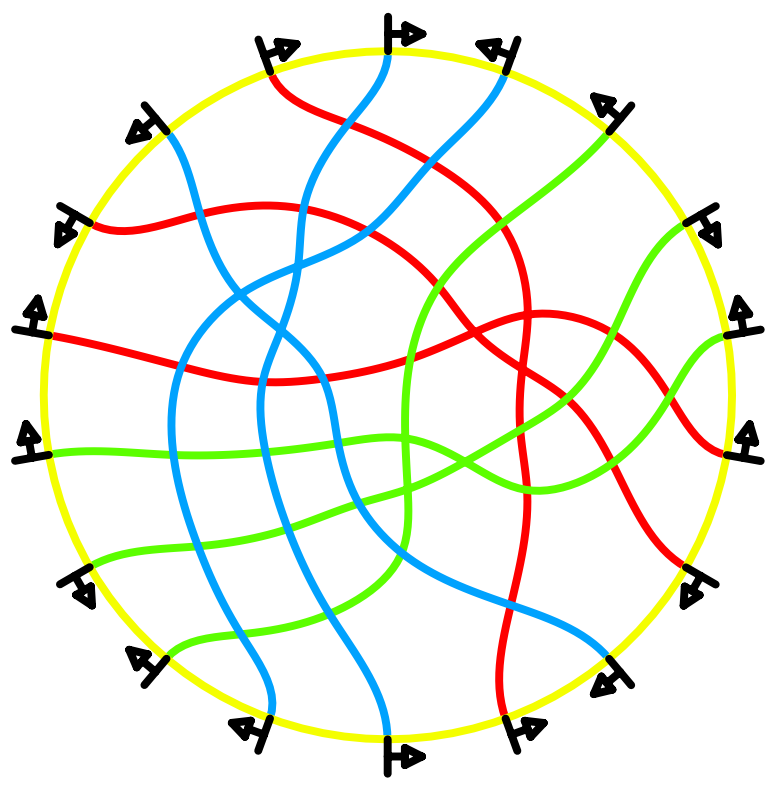

Thus we have three possible cases.

1. A triangular region versus a triangular region,

2. A 4-gon versus a 4-gon,

3. A triangular region versus a 4-gon.

In the first case, we do not have to deal with a case distinction. We can argue that we obtain the assumption for the final arguments in all cases.

For the other two cases we look at all possible corresponding pseudoline arrangements. A long and tedious but straightforward argument shows that we have all cases, up to symmetry, in Fig. 3 (we double-checked this fact with the aid of a computer and the Haskell code available at http://dino.math.unam.mx/public/code/Haskell/ OMBaranyCaratheodory.hs). We have ten types that represent Case 2 and we have eight types that represent Case 3. The lack of such a classification in higher dimensions is another obstruction to have the conjecture proved.

We now go for the main argument: pick a mutation (triangle) along the line at infinity with a red (A) and a blue (B) element for which the orientations of these elements point to the interior of that triangle.

From the corresponding figure you see, this is possible in $8+6$ cases.

We look now at the green element $(\mathrm{C})$ with the orientation such that the former $\mathrm{red} / \mathrm{blue}(\mathrm{A} / \mathrm{B})$ cocircuit lies on the negative side of $\mathrm{C}$. We have to consider 4 intersection cases of $\mathrm{C}$ with the other pseudolines $\mathrm{A}$ (red) and $\mathrm{B}$ (blue), depending on how $\mathrm{C}$ intersects $\mathrm{A}$ and $\mathrm{B}$ with respect to the cocircuit $\mathrm{A} / \mathrm{B}$.

One case is trivial. The opposite case is the one that is most involved and we look at it at the end.

The other two cases can be treated in the same way because of a symmetry argument. Let us look at such a case. We pick on the red pseudoline (A) a blue element 


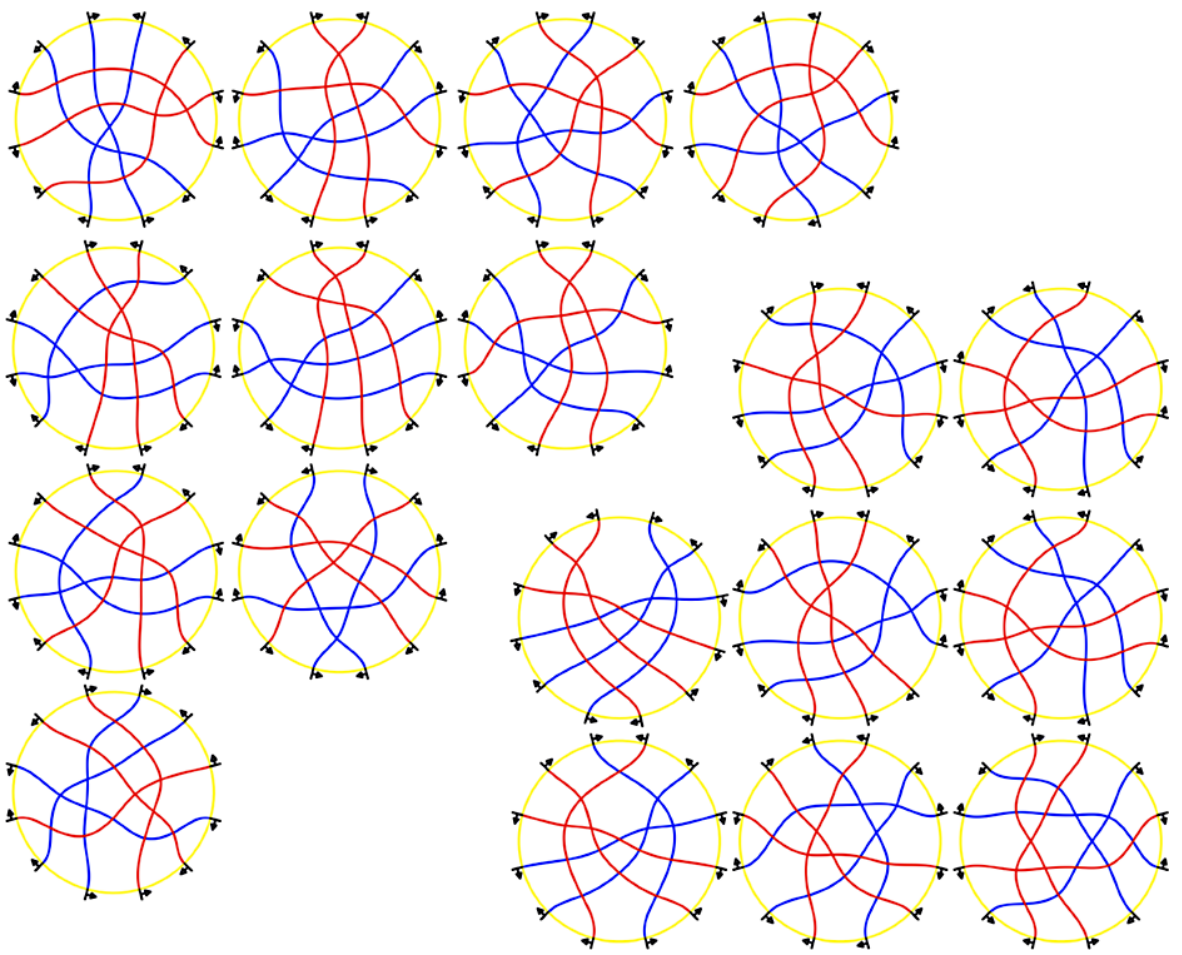

Fig. 3 (Color online) The $10+8$ cases

(D) that makes the cocircuit (A/B) negative and on the blue pseudoline (B) a red element $(\mathrm{E})$ that makes the cocircuit $(\mathrm{A} / \mathrm{B})$ negative and we see the rainbow triangles in those cases immediately, either cocircuit $A / D$ is a vertex or cocircuit $B / E$.

In the last case (of the four cases) the argument uses also those two additional pseudolines (D) and (E) and its corresponding rainbow triangles with either vertex $\mathrm{A} / \mathrm{D}$ or $\mathrm{B} / \mathrm{E}$ but in addition (when these two cases do not work!), we look at the cocircuit $(\mathrm{D} / \mathrm{E})$ that together with the green element shows us, we have a rainbow triangle in all cases.

Now observe that we did not use certain pseudolines in the above argument. For a pair of pseudolines (A, B), i.e., a (red, blue) pair, we can discard those pseudolines that have the cocircuit $\mathrm{A} / \mathrm{B}$ on the positive side.

It suffices to have a mutation along the line at infinity after deleting those pseudolines and, in the remaining cases, we can use the same argument.

\section{Remarks and Open Problems}

If instead of points we want to consider convex sets, we may use the framework of separoids [15]. A separoid is, essentially, a family of convex sets $S$ in some high dimensional Euclidian space endowed with the relation $\mid \subset\left(\begin{array}{c}2^{S} \\ 2\end{array}\right)$ defined by the (strict) 
Fig. 4 (Color online) Steinitz exchange property for Radon partitions

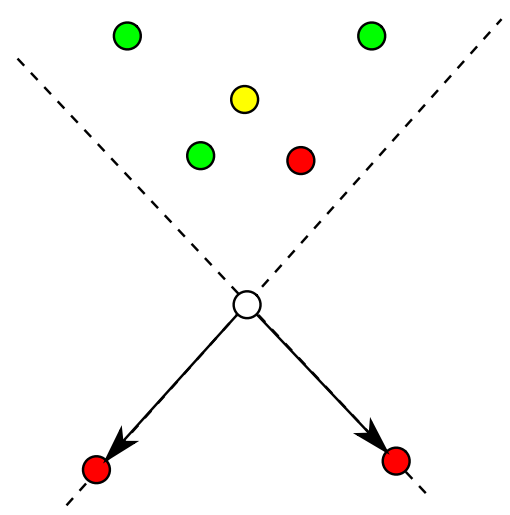

separations by hyperplanes of its subfamilies (cf. [7]); they generalise also well known combinatorial structures like hypergraphs and oriented matroids (cf. [14]).

Proposition 8 The Bárány-Carathédory theorem is true for Steinitz separoids of dimension 2.

Proof Let $S=R \cup G \cup\{\mathbf{0}\}$ be a Steinitz separoid in general position in the Euclidian plane with minimal Radon partitions $\mathbf{0} \dagger R$ and $\mathbf{0} \dagger G$, and let $\mathbf{y} \in \mathbb{E}^{2}$ be a point. Since $S$ is Steinitz, we have that there exists an $r_{y} \in R$ such that $\mathbf{0} \dagger R^{\prime}$, where $R^{\prime}=$ $R \backslash\left\{r_{y}\right\} \cup\{\mathbf{y}\}$. Again, by the Steinitz property, for each $g \in G$ there exists an $r_{g}$ such that $\mathbf{0} \dagger R_{g}^{\prime \prime}$, where $R_{g}^{\prime \prime}=R^{\prime} \backslash\left\{r_{g}\right\} \cup\{g\}$. It is easy to see that, if for each $g \in G$ we have that $r_{g}=\mathbf{y}$, then $\mathbf{0} \mid G$ which contradicts the hypothesis (see Fig. 4). Therefore, there should exists a $g \in G$ such that $R_{g}^{\prime \prime}$ is a heterochromatic triple that is not separated from $\mathbf{0}$.

This simple argument cannot be extended to higher dimensions. Indeed, it may not be true at all, as suggested by the fact that Tverberg's number for separoids is bigger than expected (cf. [13])—-more colours may be needed to guarantee the same conclusion. We pose this in the following

Problem Determine the Bárány-Carathéodory number for separoids.

A similar phenomenon occurs if the restriction is not put in the colouring of the points, but in the "position" they have. That is, if we look for all colourings of a point configuration to have, as in Theorem 2, a heterochromatic simplex capturing the origin, the planar case is different than that in higher dimensions.

Proposition 9 Let $P \subset \mathbb{E}^{2}$ be a subset in general position of the Euclidian plane. Then, for each $\{\mathbf{a}, \mathbf{b}\} \in\left(\begin{array}{c}P \\ 2\end{array}\right)$ there exists a $\mathbf{c} \in P \backslash\{\mathbf{a}, \mathbf{b}\}$ such that $\mathbf{0} \dagger \mathbf{a b c}$ if and only if for every three-colouring of $P$ there exists a heterochromatic triple $\{\mathbf{x}, \mathbf{y}, \mathbf{z}\} \in\left(\begin{array}{c}P \\ 3\end{array}\right)$ such that $\mathbf{0} \dagger \mathbf{x y z}$. 
We rephrase this result by saying: the uniform hypergraph (of rank 3) consisting of those triples $\{\mathbf{x}, \mathbf{y}, \mathbf{z}\} \in\left(\begin{array}{c}P \\ 3\end{array}\right)$ such that $\mathbf{0} \dagger \mathbf{x y z}$ is tight-its heterochromatic number equals its rank-if and only if its 2-skeleton is complete (cf. [2]).

Proof One side is clear. For the other, let $\varsigma: P \rightarrow\{r, g, b\}$ be an (effective) threecolouring of the point separoid $P$, and denote by $R=\varsigma^{-1}(r), G=\varsigma^{-1}(g)$ and $B=$ $\varsigma^{-1}(b)$ its colour classes-we speak of red, green and blue points. Further, let $\mathbf{a} \in R$ and $\mathbf{b} \in G$ be a red and a green point. By the hypothesis, there exists a $\mathbf{c} \in P$ such that $\mathbf{0} \dagger \mathbf{a b c}$; we may suppose that $\mathbf{c} \in R$ is also a red point. Since $\zeta$ is onto, there must be a blue point $\mathbf{d} \in B$ and we may suppose that it is in the cone $\operatorname{pos}(-\mathbf{a}, \mathbf{b})$; otherwise we have that either abd or bed has to be a heterochromatic triangle that is not separated from the origin. By the hypothesis, there exists a fifth point $\mathbf{e} \in P$, which we may suppose is not red, such that $\mathbf{0} \dagger$ bde. Observe that e must lie in the cone $\operatorname{pos}(\mathbf{a}, \mathbf{c})$. Then, either ade or bce is a heterochromatic triangle not separated from the origin, depending on the colour of $\mathbf{e}$ (green or blue, respectively).

Observe that any odd regular polygon centred at the origin is an example of such a tight configuration. On the other hand, in higher dimensions Proposition 9 takes the following "counterintuitive" form, which was first proved by Andreas Holmsen, János Pach and Helge Tverberg (2008) in the same paper were they proved Theorem 4:

Theorem [11] Let $P \subset \mathbb{E}^{d}$ be a finite set of points in general position, in dimension $d>2$. If $\mathbf{0} \dagger P$, then the uniform hypergraph consisting of the subsets $X \in\left(\begin{array}{c}P \\ d+1\end{array}\right)$ such that $\mathbf{0} \dagger X$ is tight if and only if $|P|=d+1$.

It is not clear yet what the correct setting is for studding all colourings of a point configuration; so far, the theorem above says that the condition on the points cannot be finite which really contrasts the content and spirit of Carathéodory's theorem. Let us pose this in the following.

Problem Characterise those-necessarily infinite-tight configurations in $\mathbb{E}^{d}$, for $d>2$.

\section{References}

1. Arocha, J.L., Bárány, I., Bracho, J., Fabila, R., Montejano, L.: Very colorful theorems. Discrete Comput. Geom. 42(2), 142-154 (2009)

2. Arocha, J.L., Bracho, J., Neumann-Lara, V.: Tight and untight triangulations of surfaces by complete graphs. J. Combin. Theory Ser. B 63(2), 185-199 (1995)

3. Bárány, I., Onn, S.: Carathéodory's theorem, colourful and applicable. In: Intuitive Geometry, Budapest, 1995. Bolyai Soc. Math. Stud., vol. 6, pp. 11-21. János Bolyai Math. Soc., Budapest (1997)

4. Bárány, I.: A generalization of Carathéodory's theorem. Discrete Math. 40(2-3), 141-152 (1982)

5. Björner, A., Las Vergnas, M., Sturmfels, B., White, N., Ziegler, G.M.: Oriented Matroids, 2nd edn. Encyclopedia of Mathematics and Its Applications, vol. 46. Cambridge University Press, Cambridge (1999)

6. Bokowski, J.G.: Computational Oriented Matroids. Cambridge University Press, Cambridge (2006). Equivalence classes of matrices within a natural framework 
7. Bracho, J., Strausz, R.: Two geometric representation theorems for separoids. Period. Math. Hung. 53(1-2), 115-120 (2006)

8. Carathéodory, C.: Über den Variabilitätsbereich der Koeffizienten von Potenzreihen, die gegebene Werte nicht annehmen. Math. Ann. 64(1), 95-115 (1907)

9. Folkman, J., Lawrence, J.: Oriented matroids. J. Combin. Theory Ser. B 25(2), 199-236 (1978)

10. Goodman, J.E.: Pseudoline arrangements. In: Handbook of Discrete and Computational Geometry. CRC Press Ser. Discrete Math. Appl., pp. 83-109. CRC Press, Boca Raton (1997)

11. Holmsen, A.F., Pach, J., Tverberg, H.: Points surrounding the origin. Combinatorica 28(6), 633-644 (2008)

12. Levi, F.W.: Die Teilung der projektiven Ebene durch Gerade und Pseudogerade. Ber. Math.-Phys. Kl. Sächs. Akad. Wiss. 78, 256-267 (1926)

13. Montellano-Ballesteros, J.J., Pór, A., Strausz, R.: Tverberg-type theorems for separoids. Discrete Comput. Geom. 35(3), 513-523 (2006)

14. Nešetřil, J., Strausz, R.: Universality of separoids. Arch. Math. (Brno) 42(1), 85-101 (2006)

15. Strausz, R.: On separoids. Ph.D. thesis, Universidad Nacional Autónoma de México (2004)

16. Strausz, R.: Erdős-Szekeres "happy end"-type theorems for separoïds. Eur. J. Combin. 29(4), 10761085 (2008) 